\title{
Prevalence of potentially inappropriate prescribing in older people in primary care and its association with hospital admission: longitudinal study
}

\author{
Teresa Pérez, ${ }^{1,2}$ Frank Moriarty, ${ }^{2}$ Emma Wallace, ${ }^{2}$ Ronald McDowell, ${ }^{2,3}$ Patrick Redmond, ${ }^{2,4}$ \\ Tom Fahey ${ }^{2}$
}

Department of Statistics and

Data Science, Complutense University of Madrid, Madrid,

Spain

${ }^{2}$ HRB Centre for Primary Care Research, Royal College of Surgeons in Ireland, Dublin, Ireland

${ }^{3}$ Cancer Epidemiology and Health Services Research

Group, Centre for Public Health, Queen's University Belfast, Belfast, UK

${ }^{4}$ Department of Public Health and Primary Care, School of Clinical Medicine, University of Cambridge, Cambridge, UK Correspondence to: F Moriarty frankmoriarty@rcsi.ie (or @FrankMoriarty on Twitter 0000-0001-9838-3625)

Additional material is published online only. To view please visit the journal online.

Cite this as: BMJ 2018;363:k4524 http://dx.doi.org/10.1136/bmj.k4524

Accepted: 15 October 2018

\section{ABSTRACT}

OBJECTIVE

To determine whether hospital admission is associated with potentially inappropriate prescribing among older primary care patients (aged $\geq 65$ years) and whether such prescribing was more likely after hospital admission than before.

DESIGN

Longitudinal study of retrospectively extracted data from general practice records.

SETTING

44 general practices in Ireland in 2012-15.

PARTICIPANTS

Adults aged 65 years or over attending participating practices.

\section{EXPOSURE}

Admission to hospital (any hospital admission versus none, and post-admission versus pre-admission).

\section{MAIN OUTCOME MEASURES}

Prevalence of potentially inappropriate prescribing assessed using 45 criteria from the Screening Tool for Older Persons' Prescription (STOPP) version 2 , analysed both as rate of distinct potentially inappropriate prescribing criteria met (stratified Cox regression) and binary presence of potentially inappropriate prescribing (logistic regression) and adjusted for patients' characteristics. A sensitivity analysis used matching with propensity scores based on patients' characteristics and diagnoses.

RESULTS

Overall 38229 patients were included, and during 2012 the mean age was 76.8 (SD 8.2) years and

\section{WHAT IS ALREADY KNOWN ON THIS TOPIC}

Potentially inappropriate prescribing is common among older people

It is associated with adverse outcomes including emergency hospital attendances and admissions, adverse drug events, and poorer quality of life Research to date has focused on characteristics of patients and general practitioners as risk factors for poor prescribing quality

\section{WHAT THIS STUDY ADDS}

Hospital admission was independently associated with an increased rate of potentially inappropriate prescriptions

Patients who were admitted to hospital were more likely to have potentially inappropriate prescribing after admission compared with before, independent of patients' characteristics

This illustrates the need to consider and overcome potential adverse effects of hospital admission on appropriateness of prescribing among older patients

43\% (13 212) were male. Each year, 10.4-15.0\% (3015/29077 in 2015 to 4537/30 231 in 2014) of patients had at least one hospital admission. The overall prevalence of potentially inappropriate prescribing ranged from $45.3 \%$ (13940/30 789) of patients in 2012 to $51.0 \%$ (14 823/29 077) in 2015. Independently of age, sex, number of prescription items, comorbidity, and health cover, hospital admission was associated with a higher rate of distinct potentially inappropriate prescribing criteria met; the adjusted hazard ratio for hospital admission was 1.24 (95\% confidence interval 1.20 to 1.28 ). Among participants who were admitted to hospital, the likelihood of potentially inappropriate prescribing after admission was higher than before admission, independent of patients' characteristics; the adjusted odds ratio for after hospital admission was 1.72 (1.63 to 1.84). Analysis of propensity score matched pairs showed a slight reduction in the hazard ratio for hospital admission to 1.22 (1.18 to 1.25$)$.

\section{CONCLUSION}

Hospital admission was independently associated with potentially inappropriate prescribing. It is important to determine how hospital admission may affect appropriateness of prescribing for older people and how potential adverse consequences of admission can be minimised.

\section{Introduction}

Adults aged 65 years and over are a growing population and represent the largest consumers of prescribed drugs. ${ }^{12}$ Although optimal prescribing aims to maximise benefits to patients while minimising harms and costs, achieving this balance when caring for older patients in primary care can be challenging. Physiological changes in ageing can impair metabolism and excretion of drugs and increase sensitivity to their effects. ${ }^{3}$ In addition, older patients tend to have a higher burden of multimorbidity and so take more drugs, contributing to both increased treatment burden and potential drug-drug and drug-disease interactions. ${ }^{4}$ Lastly, although most prescribing in primary care is repeat prescribing, ${ }^{5}$ such drugs are often initially prescribed in secondary care, which can be problematic as the general practitioner is responsible for coordination and managing all prescriptions. ${ }^{6}$ This can be even more challenging for patients with multimorbidity who attend multiple healthcare providers.

Use of prescribed drugs among older adults is increasing despite the high risk of adverse drug events and resultant morbidity and mortality. ${ }^{127} \mathrm{~A}$ recent 
systematic review focusing on adverse drug events in ambulatory care found prevalence rates ranging from $2.8 \%$ to $34.7 \%$, up to a quarter of which were judged to be preventable. ${ }^{8}$ Another systematic review reported that $9.9 \%$ of all hospital admissions in people aged 65 years or over were as a result of an adverse drug event. ${ }^{9}$

Appropriateness of prescribing can be assessed by process measures (that is, what providers do) or outcome measures (that is, patient outcomes). These measures can be either implicit (judgment based) or, more often, explicit (criterion based). ${ }^{10}$ Examples of explicit measures include the Beers criteria and the Screening Tool of Older Person's potentially inappropriate Prescribing (STOPP) and Screening Tool to Alert doctors to the Right Treatment (START). ${ }^{11}$ Explicit measures have the advantage of being based on literature review and expert consensus, and they are reliable and have content validity, although they do periodically need revision to reflect new evidence. In 2015 the STOPP/START criteria were updated to add new criteria and remove obsolete ones. ${ }^{11}$ In STOPP/ START 2, the final list of 114 criteria, including 80 STOPP criteria and 34 START criteria, was agreed after two rounds of Delphi validation. ${ }^{11}$ The STOPP/ START 2 criteria can be used to examine potentially inappropriate prescribing in older people.

The adverse outcomes associated with the STOPP criteria are well established, including adverse drug events, emergency admissions or emergency department visits, and poorer quality of life. ${ }^{12-15}$ Previous studies have examined predictors of potentially inappropriate prescribing, such as patients' characteristics (for example, multimorbidity, age, and number of prescribed drugs), and characteristics of general practices (for example, deprivation of catchment area). ${ }^{1617}$ There has been less focus on how health system factors, such as hospital admission or care transitions, may contribute to the appropriateness of prescribing for ambulatory care patients.

Therefore, the objectives of this study were to use the revised STOPP criteria to estimate the annual prevalence of potentially inappropriate prescribing in older community dwelling people in Ireland, to examine any association between hospital admission and potentially inappropriate prescribing, and to compare the prevalence of potentially inappropriate prescribing before and after hospital admission. We hypothesised that occurrence of potentially inappropriate prescribing among older adults may be significantly associated with hospital admission and, among patients who were admitted to hospital, occurrence of potentially inappropriate prescribing may differ before and after admission.

\section{Methods}

\section{Study population and study design}

This was a longitudinal study using a retrospectively collected dataset that included general practitioners' patients aged 65 years or over between 2012 and 2015. We used the patient management system Socrates (www.socrates.ie) to collect data from 44 general practices in Ireland, including prescribing, demographic, clinical, and hospital admission records. Socrates is one of four electronic health record vendor systems accredited by the Irish College of General Practitioners. Most (94\%) general practices in Ireland are computerised, and electronic morbidity coding and prescribing occurs in more than $90 \%$ of these computerised practices. ${ }^{18}$ Although the validity of morbidity recording in Ireland is not as good as in the UK, recent initiatives have improved both completeness and validity of morbidity coding. ${ }^{19}$ Socrates has created quality indicator tools used for audit and also in research, such as a study of resistance patterns of urinary tract infections. ${ }^{20}$ The STROBE (STrengthening the Reporting of OBservational studies in Epidemiology) statement was used in the conduct and reporting of this study. ${ }^{21}$

\section{Explanatory variables and outcomes of interest}

We identified potentially inappropriate prescribing, according to 45 STOPP 2 criteria, by using information on drugs and diagnoses for each patient in the dataset, in each of the four years. A total of 35 (44\%) criteria could not be applied-for example, owing to lack of information on laboratory monitoring, history of falls, or prescribing indication (see appendix 1). Where necessary, we included prescribing and diagnosis information from before 2012 when estimating the prevalence of potentially inappropriate prescribing-for example, for criteria relating to first line treatment. An extensive description of criteria and their application is provided in appendix 2. For each patient, we calculated the total number and dates of first occurrence of distinct potentially inappropriate prescribing criteria met per calendar year. We analysed these either as recurrent events (that is, rate of distinct potentially inappropriate prescribing criteria met per year) or as a dichotomous variable (at least one potentially inappropriate prescription in the period considered or no event).

The STOPP criteria, as explicit measures of inappropriate prescribing, have been used extensively in research as measures of the process of care. Their validity has been established in multiple studies showing their relation with important outcomes for patients. In terms of predictive validity, STOPP modestly discriminates for outcomes such as adverse drug events, emergency department visits, and hospital admissions (C indices of 0.65-0.70). ${ }^{22}$ Other observational studies have found consistent associations between the STOPP criteria and avoidable adverse drug events relevant to the index admission among hospital inpatients, ${ }^{12}$ poorer quality of life, ${ }^{14}$ emergency department visits, ${ }^{14}$ and unplanned readmission to hospital. ${ }^{23}$ Prescribing included in STOPP was considered causal in 30\% of adverse drug reactions in a Swedish study in an older population, ${ }^{24}$ and in a study of definitely or possibly avoidable adverse drug events that led to hospital admission, $62.2 \%$ were listed in the STOPP criteria. ${ }^{12}$ On this basis, the STOPP criteria can be considered a valid process 
measure of quality of care, and they have been used as primary outcomes in interventional trials aiming to improve prescribing. ${ }^{25} 26$

To examine the association between hospital admission and potentially inappropriate prescribing, we defined the explanatory variable hospital admission as a dichotomous variable (no hospital admission versus any admission). Hospital admission was incorporated as a time dependent variable in the Cox model, considered as "no hospital admission" before the date of the first admission and "hospital admission" after that date. All practices included elective and emergency admissions to public hospitals, and four of the 44 practices additionally included emergency department attendances. For the comparison of potentially inappropriate prescribing before and after hospital admission, the explanatory variable was time period (after hospital admission versus before). The post-admission period started on the day after hospital admission. For those patients admitted more than once in the same year, we considered only the first admission.

Categorical covariates adjusted for in all models were sex and type of health cover (with four categories: General Medical Services (GMS) scheme, Doctor Visit Card (DVC), private patient, and other). Continuous covariates were age (years), number of prescription items in that period, and multimorbidity. The GMS and DVC schemes are types of public health coverage, providing eligible patients with a range of health services including general practitioner visits free of charge. These are means tested, with eligibility based on household income and age. The GMS scheme covers the most socioeconomically deprived people, approximately one third of the population, and 90\% of those aged 70 years or over, for whom a lower income threshold applies. ${ }^{27}$ The DVC scheme covers people with higher, but still limited, means. Other people pay out-of-pocket for primary care services such as general practitioner visits and drugs; hence Ireland has a mixed public-private healthcare system. We assessed the number of prescription items as the total number of items prescribed to a patient per year, not accounting for multiple issues/repeats on prescriptions. We assessed multimorbidity by using the Charlson comorbidity index. ${ }^{28}$ This index is based on 17 conditions weighted by one year mortality risk, and a higher score indicates more severe comorbidity.

\section{Statistical analyses \\ Annual prevalence of potentially inappropriate prescribing}

We described demographic and clinical characteristics of patients (such as age, sex, health cover type, number of prescription items, and multimorbidity) and the overall prevalence of potentially inappropriate prescribing for each year considered. Data are expressed as mean (standard deviation), median (interquartile range), and proportions (absolute and relative frequencies) as appropriate. Analyses were run on a complete case basis, and the numbers of people included in each analysis are reported in the relevant tables and figures.

\section{Association between potentially inappropriate prescribing and hospital admission}

We examined the relation between potentially inappropriate prescribing and hospital admission adjusted for age, sex, health cover type, number of prescription items, and multimorbidity. We fitted both a mixed effect logistic regression model (in which the outcome was defined as dichotomous ( 0 without any potentially inappropriate prescribing in that year, or 1 otherwise)) and the Prentice, Williams, and Peterson (PWP) model (in which the outcome was time from the beginning of the year to a new potentially inappropriate prescribing criterion observed in each patient per calendar year). The mixed effect logistic regression model extends the general linear model by incorporating correlations among the outcomes (multiple observations per patient). This can be accomplished by including random effects. In this study, we introduced two random effects representing the patient and the year. We used the MCMCglmm package in $\mathrm{R}^{29}{ }^{30}$ because models obtained using the glmer function of the lme4 package did not converge. Modelling of the rate of distinct potentially inappropriate prescribing events used the PWP model, ${ }^{31}$ which is an extension of the Cox proportional hazard model. We used a stratum variable to keep track of the number of previous potentially inappropriate prescribing criteria met, allowing the hazard for a new potentially inappropriate prescribing criterion to change after a previous event. We used a robust variance estimator to account for individual patients' heterogeneity. ${ }^{32}$ We included hospital admission as a time dependent variable that could change from "no hospital admission" to "hospital admission" during each year. We did a stratified analysis for health cover type because the proportional hazard assumption was not satisfied. We obtained an overall hazard ratio for the whole study period, also stratifying by year. As the date of death was not included in the dataset, within each year the length of follow-up was until the end of the year if the patient had a record in the following year or up until the date of the last prescription in that year if not. We used the survival package in $\mathrm{R}$ for this analysis. ${ }^{33}$ To avoid double counting, we omitted criterion 32 from this analysis because it overlapped fully with criterion 1 (both relate to long term use of non-steroidal anti-inflammatory drugs; see appendix 2 for further details).

\section{Potentially inappropriate prescribing before and after admission to hospital}

We did a second analysis comparing potentially inappropriate prescribing before and after hospital admission among only those patients who were admitted to hospital during a study year. Paired sample tests (that is, having two observations per patient: one for presence/absence of potentially inappropriate prescribing before hospital admission 
and one for after admission) allowed the temporality of the relation between hospital admission and potentially inappropriate prescribing to be assessed and also accounted for between patient variability. We fitted a mixed effect logistic regression model and included a random intercept for each patient to allow between patient variability in the outcome and for each year, using the MCMCglmm package in . $^{30}$ The outcome was whether or not the patient had any potentially inappropriate prescribing event in the time period considered. The explanatory variable was time period (after hospital admission, relative to before admission), with adjustments made for the covariates listed above. In all analyses, we defined $\mathrm{P}<0.05$ as statistically significant.

\section{Sensitivity analyses}

Firstly, we repeated each of the above analyses separately by calendar year to assess the consistency of observed associations over the study period. Secondly, owing to some missing data for the Charlson comorbidity index, we also repeated analyses using an alternative measure of multimorbidity. We used RxRisk-V, a prescription based measure of morbidity including medication proxies for 45 conditions, which has shown criterion validity and reliability compared with patients' medical diagnoses. ${ }^{34}$ Prescription data were available for all included participants, and RxRisk was adjusted for in models as a binary indicator of multimorbidity (that is, two or more conditions).

Lastly, as patients were not randomly allocated to being admitted to hospital or not, these groups may have differences in their characteristics that could bias estimates. We did a sensitivity analysis using propensity score matching to assess whether the association between hospital admission and potentially inappropriate prescribing could be due to unmeasured confounders. ${ }^{35}$ We used the propensity score, defined as the conditional probability of hospital admission given the measured covariates, to balance covariates in the two groups. Using the MatchIt package in $\mathrm{R},{ }^{36}$ we first fitted a logistic regression model to estimate propensity scores. We modelled the conditional probability of hospital admission as a function of baseline and those clinical characteristics associated with admission that were also independent risk factors for potentially inappropriate prescribing. These variables included age, sex, health cover type, number of prescription items, Charlson comorbidity index, and whether the patient had been diagnosed as having any of the five most common conditions (diabetes, chronic obstructive pulmonary disease, any type of tumour, a myocardial infraction, or cerebrovascular disease). We randomly selected each patient with a hospital admission and then matched them with the patient with no admission with the closest propensity score. Finally, we fitted the same models considering only the matched pairs.

\section{Patient involvement}

Patients were not involved in the conception, design, or conduct of this research. We plan to disseminate the findings to the public and patients through our contacts in patient representative bodies, the popular media, and the participating general practices.

\section{Results}

\section{Descriptive statistics}

A total of 38229 patients were included in the dataset over the period 2012 to 2015 . Table 1 shows demographics and clinical characteristics of this sample, by year. We excluded patients without prescriptions during the period analysed. During 2012, the mean age of included patients was 76.8 (SD 8.2) years and $43 \%$ were male. For each study year, 10.4$15.0 \%$ of patients had at least one hospital admission.

\section{Annual prevalence of potentially inappropriate prescribing}

The overall prevalence of potentially inappropriate prescribing ranged from 45.3\% (13940/30789) of patients in 2012 to $51.0 \%(14823 / 29077)$ in 2015 (appendix 3). The individual criteria with the highest prevalence in 2015 included proton pump inhibitor for uncomplicated peptic ulcer disease or erosive peptic oesophagitis at full therapeutic dosage for more than eight weeks $(7836 ; 26.9 \%)$, benzodiazepines for at least four weeks $(5562 ; 19.1 \%)$, and drugs prescribed beyond the recommended duration (3988; 13.7\%, primarily driven by $\mathrm{Z}$ drug hypnotics (zolpidem, and

\begin{tabular}{|c|c|c|c|c|c|}
\hline Demographic and clinical characteristics & $2012(n=30753)$ & $2013(n=30789)$ & $2014(n=30231)$ & $2015(n=29077)$ & Missing data (\%) \\
\hline Mean (SD) age, years & $76.8(8.2)$ & $76.4(8.1)$ & $75.9(7.8)$ & $75.0(7.6)$ & 0.08 \\
\hline Male sex & $13212(43.0)$ & $13335(43.3)$ & $13176(43.6)$ & $12687(43.6)$ & 0.08 \\
\hline Patients with hospital admission & $4151(13.5)$ & $4496(14.6)$ & $4537(15.0)$ & $3015(10.4)$ & 0 \\
\hline \multicolumn{5}{|l|}{ Health cover: } & \multirow{5}{*}{0.03} \\
\hline General Medical Services scheme & $21053(68.5)$ & $21472(69.7)$ & $21202(70.1)$ & $20859(71.7)$ & \\
\hline Doctor Visit Card & $3029(9.8)$ & $3153(10.2)$ & $3201(10.6)$ & $3280(11.3)$ & \\
\hline Private patients & $6518(21.2)$ & $6004(19.5)$ & $5705(18.9)$ & $4817(16.6)$ & \\
\hline Other & $153(0.5)$ & $160(0.5)$ & $123(0.4)$ & $71(0.2)$ & \\
\hline Median (interquartile range) prescription items per patient & $22(9-42)$ & $22(9-43)$ & $23(10-44)$ & $21(9-40)$ & 0 \\
\hline Mean (SD) Charlson comorbidity index & $0.89(1.23)$ & $0.94(1.27)$ & $1(1.31)$ & $1(1.31)$ & 24.2 \\
\hline \multicolumn{5}{|l|}{ Prevalence of potentially inappropriate prescribing events: } & \multirow{4}{*}{0} \\
\hline 1 & $6452(21.0)$ & $6843(22.2)$ & $6771(22.4)$ & $6857(23.6)$ & \\
\hline 2 & $4171(13.6)$ & $4254(13.8)$ & $4429(14.6)$ & $4220(14.5)$ & \\
\hline$\geq 3$ & $3317(10.8)$ & $3654(11.9)$ & $3762(12.4)$ & $3746(12.9)$ & \\
\hline
\end{tabular}


zopiclone) for more than four weeks), and this was observed in each calendar year (appendix 3).

\section{Association between potentially inappropriate prescribing and hospital admission}

In the PWP regression model, hospital admission, higher age, greater number of prescription items, and multimorbidity were all associated with a higher rate of potentially inappropriate prescribing events. The rate of distinct criteria met per year increased by $24 \%$ if a patient had been admitted to hospital (hazard ratio $1.24,95 \%$ confidence interval 1.20 to 1.28 ) when controlled for the other covariates (fig 1). For sex, the rate of potentially inappropriate prescribing criteria met per year was approximately $12 \%$ lower for men (hazard ratio $0.88,0.87$ to 0.89 ). The rate of distinct potentially inappropriate prescribing criteria observed in one year also increased as age, number of prescription items, and multimorbidity increased.

Results obtained from the mixed effect logistic regression model were analogous, although in this model age was not significant (see appendix 4). The odds ratio for hospital admission was 1.49 (1.42 to 1.58) - that is, the probability of at least one potentially inappropriate prescription during a year increased by $49 \%$ for patients admitted to hospital, after adjustment for relevant covariates.

\section{Potentially inappropriate prescribing before and after admission to hospital}

Having analysed potentially inappropriate prescribing in patients who were admitted to hospital compared with those who were not, we determined the effect of admission on a patient's likelihood of having potentially inappropriate prescribing among only those patients who were admitted. Figure 2 shows the estimated odds ratios with $95 \%$ credible intervals. Among patients who had at least one hospital admission in a year, the risk of having any potentially inappropriate prescription increased by $72 \%$ after admission to hospital. Women and patients with greater numbers of prescription items were more likely to have potentially inappropriate prescriptions.

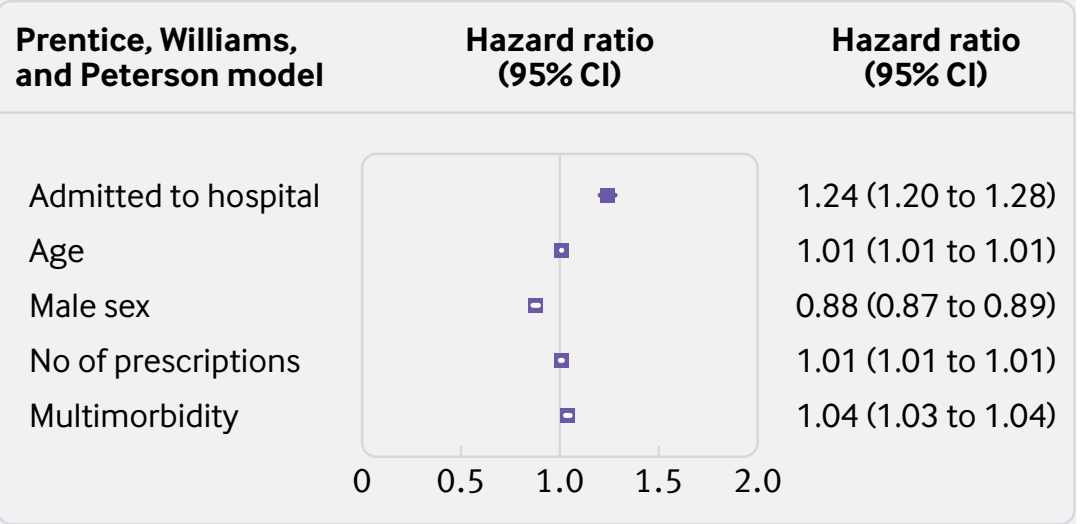

Fig 1 | Estimated hazard ratios $(95 \% \mathrm{Cl})$ for rate of distinct potentially inappropriate prescribing criteria met among all participants. Reference groups were no hospital admission and female sex

\section{Sensitivity analysis}

When analyses were repeated on a year by year basis, the relation between hospital admission and potentially inappropriate prescribing was consistent over time (appendix 5). Adjustment for multimorbidity using RxRisk instead of the Charlson comorbidity index (table 2), and therefore inclusion of participants for whom diagnostic coding may have been missing, resulted in little change in the magnitude of the parameter estimates for hospital admission. Lastly, propensity score matching compared patients admitted to hospital with those who were not admitted, using both the PWP model (fig 3) and the logistic model (appendix 6). These analyses still showed a statistically significant association between hospital admission and the outcome of potentially inappropriate prescribing (adjusted hazard ratio 1.22, 1.18 to 1.25 ; adjusted odds ratio $1.48,1.37$ to 1.58 ).

\section{Discussion}

This study found that a substantial proportion of community dwelling older people had at least one potentially inappropriate prescription defined according to the STOPP 2 criteria and that hospital admission was a significant marker of potentially inappropriate prescribing. Setagainstageneral increase in potentially inappropriate prescribing and patients with multiple potentially inappropriate prescribing criteria met, we determined that after control for the characteristics assessed in this study (such as age, number of prescriptions, and multimorbidity) hospital admission was associated with a higher rate of potentially inappropriate prescribing. Furthermore, for patients who were admitted to hospital, their likelihood of having potentially inappropriate prescribing increased by $72 \%$ after admission compared with before, independent of other patient related factors. These relations were consistent across study years and were robust to different analytical approaches in sensitivity analyses.

\section{Strengths and weaknesses of study}

This study included a large sample of community dwelling older adults and used the most recent version of the STOPP criteria to assess potentially inappropriate prescribing. Using two different approaches (unpaired and paired samples), we obtained consistent conclusions. However, owing to the secondary nature of this analysis, 35 (44\%) of 80 STOPP criteria for which relevant patient information was unavailable could not be applied (see appendix 1). Like other explicit measures of potentially inappropriate prescribing, STOPP does not account for clinical judgment and individual clinical circumstances in which prescribing may be justified and appropriate in certain patients. However, STOPP has consistently been associated with poorer patient outcomes. ${ }^{13}$ The quality of clinical coding of diagnoses was somewhat variable, which precluded application of the START criteria to identify prescribing omissions. This may be of 


\begin{tabular}{|c|c|c|}
\hline Logistic model & $\begin{array}{l}\text { Odds ratio } \\
(95 \% \mathrm{Cl})\end{array}$ & $\begin{array}{l}\text { Odds ratio } \\
(95 \% \mathrm{Cl})\end{array}$ \\
\hline After hospital admission & \multirow[b]{2}{*}{ a } & $1.72(1.63$ to 1.84$)$ \\
\hline Age & & $1.00(1.00$ to 1.01$)$ \\
\hline Male sex & $=$ & 0.81 (0.76 to 0.85$)$ \\
\hline No of prescriptions & - & 1.03 (1.03 to 1.03$)$ \\
\hline Multimorbidity & $=$ & 1.01 (0.98 to 1.02$)$ \\
\hline & $\begin{array}{lll}0.5 & 1.0 & 1.5\end{array}$ & \\
\hline
\end{tabular}

Fig 2 | Estimated odds ratios (with $95 \%$ credible intervals) for presence of potentially inappropriate prescribing among only participants admitted to hospital. Reference groups were before hospital admission and female sex. Also adjusted for patient health cover type, which did not show any significant association

particular interest for future research assessing the effect of hospital admission on appropriateness of prescribing, as unintentional omission of treatments is noted as the most common medication error at transitions of care. ${ }^{37}$

We addressed the quality of clinical and diagnostic coding by doing a sensitivity analysis using a prescription based measure of multimorbidity for adjustment and showed little effect on the results. Although general practices were recruited from a wide geographical area, they may not be representative of all practices, with the potential for volunteer bias. However, the analysis included patients with any type of health cover, compared with other studies limited to participants eligible for the means tested GMS scheme. Variability in coding for hospital admission existed between practices, and private hospital admissions were not captured, leading to potential for misclassification of exposure status for patients. However, the vast majority of secondary care interactions for older patients would be with public hospitals, so this is unlikely to significantly affect the findings. A high percentage of patients had complete follow-up over the course of the study. We did not have

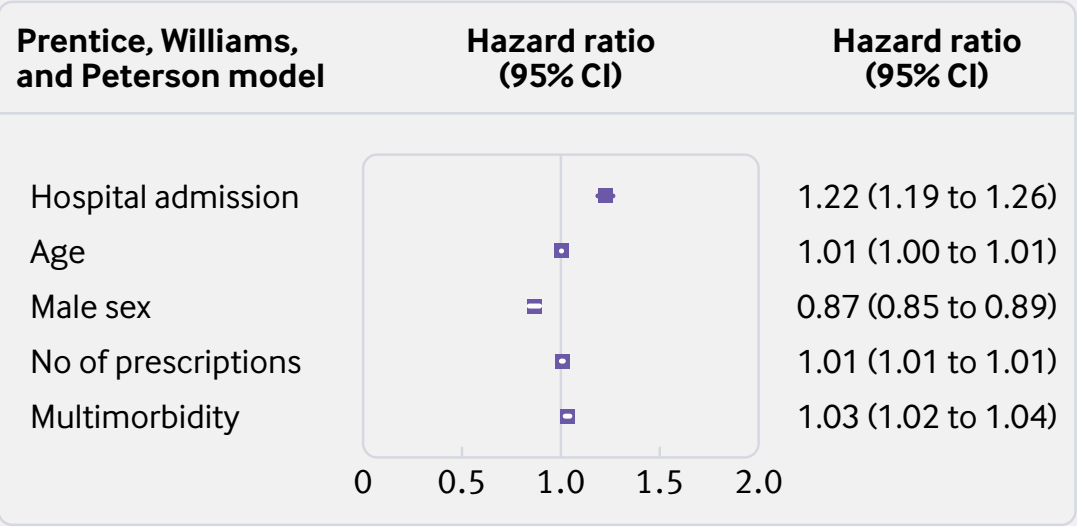

Fig 3 | Estimated hazard ratios $(95 \% \mathrm{Cl})$ for rate of distinct potentially inappropriate prescribing events among propensity score matched participants. Reference groups were no hospital admission and female sex access to reasons for loss to follow-up, which could include mortality, moving practice, or admission to care homes (residential or nursing). However, results were robust to the possibility of dependent censoring. Although we adjusted for a range of characteristics of patients, as with any observational study potential exists for unmeasured confounding, which may partly or fully explain the relation between hospital admission and potentially inappropriate prescribing. We assessed the robustness of our result to different adjustment methods by using a propensity matched sensitivity analysis, but residual confounding due to other factors, such as the severity of illness, may still exist.

\section{Comparison with previous studies}

The literature examining the effect of hospital admission on potentially inappropriate prescribing is limited. Some studies have compared medication appropriateness at hospital admission and discharge, including potentially inappropriate prescribing defined by Beers criteria alone or in addition to STOPP/ START. $^{38-40}$ In these studies, either no difference or a small reduction in potentially inappropriate prescribing was found between admission and discharge. ${ }^{38-40}$ However, only the relatively short period of hospital admission was considered and the effect on primary care prescribing after discharge was not assessed. These studies included between approximately 180 and 2000 patients; in contrast to our study of more than 40000 people, they may have been underpowered to detect an association.

A previous study assessed the prevalence of potentially inappropriate prescribing among 1016 older GMS scheme patients in Ireland presenting at one emergency department after a fall. ${ }^{41}$ The overall prevalence of both the STOPP criteria (version 1) and Beers criteria (2012) did not change in the 12 months after the fall compared with before the fall. Prescribing of some drugs associated with falls, such as neuroleptics and benzodiazepines, did decrease, however. Discordance between these findings and ours may be because these patients were attending hospital for a specific adverse event (a fall), so an assessment of risk factors contributing to this, including drugs, was likely done during or after discharge from hospital.

In our study, we applied the recently revised 2015 STOPP 2 criteria - that is, the most current definition of potentially inappropriate prescribing. The prevalence here is closely comparable to estimates from other studies using STOPP 2, which ranged from $40.4 \%$ and $56 \%$ among community dwelling people aged at least 65 and 80 years, respectively, ${ }^{42}{ }^{43}$ to between $41.5 \%$ and $71.5 \%$ in older patients being discharged from hospital. ${ }^{3944}$ As in our study, long term prescription of benzodiazepines and $\mathrm{Z}$ drugs was common in several other studies using STOPP 2. ${ }^{39} 42-45$ In contrast, the long term use of proton pump inhibitors, the most common criterion in our study, was noted as particularly prevalent in only two previous studies using STOPP 2. ${ }^{3945}$ 


\begin{tabular}{|c|c|c|c|}
\hline Estimate & No & Hazard ratio ( $95 \%$ confidence interval)* & Odds ratio ( $95 \%$ credible interval)* \\
\hline \multicolumn{4}{|c|}{ Admitted to hospital (relative to not admitted) } \\
\hline Adjusted for Charlson comorbidity index & 28831 & $1.24(1.20$ to 1.27$)$ & $1.49(1.42$ to 1.59$)$ \\
\hline Adjusted for RxRisk & 38169 & $1.25(1.22$ to 1.29$)$ & $1.55(1.47$ to 1.64$)$ \\
\hline \multicolumn{4}{|c|}{ After admission (relative to before admission) } \\
\hline Adjusted for Charlson comorbidity index & 9549 & - & $1.72(1.63$ to 1.84$)$ \\
\hline Adjusted for RxRisk & 11277 & - & $1.71(1.63$ to 1.81$)$ \\
\hline
\end{tabular}

Implications for clinicians, research, and policy Inpatient admissions can provide the opportunity for specialist teams to review and optimise management of older patients' chronic conditions, including their drugs. ${ }^{46}$ Although hospital admissions have the potential to improve management of drugs, this study suggests these possible benefits to appropriateness of prescribing after discharge to primary care are not being realised. Our findings suggest that hospital admission (which may result from a change in a patient's clinical status and may result in an intensification of healthcare) is an important driver of potentially inappropriate prescribing and the overuse and/or misuse of drugs. Medicines management services for inpatients in Ireland are broadly similar to those in the UK; however, the extent to which they are provided in practice is variable owing to resourcing of hospital pharmacy services. In approximately $40 \%$ of Irish hospitals, pharmacists do admission medication reconciliation and review, which is similar to the proportion in UK hospitals, although fewer Irish hospitals involve pharmacists in emergency department and acute medical assessment units. ${ }^{47}$ Most provide inpatient clinical pharmacy services; however, unlike the UK, in most (86\%) Irish hospitals pharmacists had no formal involvement in the discharge prescribing process. The vast majority also do not supply drugs to patients on discharge, and about half provide pharmacist counselling on discharge drugs. ${ }^{47}$ The 2017 National Patient Experience Survey report underlines the need for improved medication management services at discharge, where $40 \%$ of patients reported not being advised about drug side effects to be aware of. ${ }^{48}$

Poor coordination of transitions between care settings (from secondary to primary care), can put patients at increased risk of medication errors, adverse drug events, and readmissions. ${ }^{49-51}$ Improving coordination of care, particularly for older patients with complex care needs, has been identified as an international policy priority. ${ }^{52}$ Transitional care interventions for older patients with chronic disease discharged from hospital to primary care have been evaluated in a recent systematic review. ${ }^{54}$ Evidence suggests that these interventions can reduce mortality, hospital readmissions, and number of readmission days after 3-18 months (for example, a mortality risk difference at 18 months of -0.07 (95\% confidence interval -0.12 to -0.02$)$ ), but no evidence of a benefit to quality of life was shown in meta-analysis. ${ }^{54} \mathrm{~A}$ recent quasi-experimental study evaluated the effect of a medication management system (Pharm2Pharm) provided by hospital and community pharmacists for older adults at risk of medication problems. ${ }^{55}$ The intervention seemed to reduce the drug related hospital admission rate and provide cost savings.

More effective means of medicines reconciliation in hospital and primary care-for example, through the availability of a summary care record-may allow for more of clinicians' time to be focused on assessment of the appropriateness of drugs. ${ }^{56}$ Similarly, implementing a standardised electronic format for discharge summaries could improve their quality and reduce discrepancies arising from transitions between hospital and primary care. ${ }^{57}$ As well as reducing deficits in communication, a robust electronic record system could also incorporate decision support to aid clinicians in reviewing prescriptions, which, combined with incentives and professional education, has been shown to effectively reduce high risk prescribing and associated adverse events. ${ }^{58}$ A large scale study of almost a million patients in UK general practice showed high variation between practices in the prevalence of such high risk prescribing, ${ }^{59}$ suggesting that practice level interventions to improve prescribing should be targeted. Variation among practices in the effect of hospital admission on appropriate prescribing also warrants examination to help to inform strategies to improve this.

Individual clinicians may consider several potential solutions. A recent systematic review identified incomplete clinical picture (information deficits due to poor communication among multiple prescribers and fragmentation at care interfaces) as a barrier to minimising inappropriate drugs by prescribers. $^{60}$ Many of the common STOPP criteria in our study relate to inappropriate duration of use, so documenting and clearly communicating the intended duration of the prescription or a planned review date would ensure that other clinicians such as general practitioners have complete information for reviewing and stopping such prescriptions. Similarly, documentation of the indication for a drug will facilitate review of appropriateness and continued need. ${ }^{60}$ The indication and duration should also be discussed with patients, which would mean that they expect future review or stopping of drugs and thus reduce the ambivalence/resistance of patients to change as a barrier to appropriate prescribing. ${ }^{6061}$ Prescribers have also cited a lack of evidence and difficulty in assessing the benefits/harms of treatment 
as a barrier. ${ }^{60}$ Several evidence based guidelines have recently been developed to support decisions on deprescribing specific drugs, including proton pump inhibitors, benzodiazepines, and Z drugs, which were among the most prevalent problems identified in our study. ${ }^{62}{ }^{63}$ Deprescribing algorithms and patient information leaflets and decision aids as companions to these guidelines are also available from www. deprescribing.org.

We cannot determine whether the observed increase in potentially inappropriate prescribing is a consequence of illness that prompted hospital admission, and the increased complexity this may bring, or whether potentially inappropriate prescribing is a consequence of further medical intervention during the hospital stay. Future research should identify the mechanisms by which hospital admission is associated with potentially inappropriate prescribing, including detailed review of patients' clinical records to explore how potentially inappropriate prescribing may have been contributory or causal in hospital admissions and to understand the clinical decisions (in both primary and secondary care) that resulted in potentially inappropriate prescribing among patients after discharge from hospital. Research should also evaluate how to overcome these problems to enhanceappropriateness of prescribing for older patients after discharge. This may include better continuity of information through improved health information and communication technology infrastructure, as well as formal transitional care programmes. ${ }^{54}$ In addition, hospital based interventions to enhance appropriateness of prescribing for older patients should be evaluated, such as reviews using prescribing criteria like STOPP or alignment of clinical pharmacists with medical teams to provide integrated medicines management. ${ }^{6566}$

\section{Conclusions}

This study shows that potentially inappropriate prescribing is becoming increasingly prevalent among community dwelling older people according to the most recent STOPP criteria. Furthermore, hospital admission is independently associated with an increased risk of potentially inappropriate prescribing after discharge back to primary care. Identifying optimal management strategies for older people is vital to ensure that the risk of inappropriate drugs is minimised after transitions of care.

We gratefully acknowledge the contributions of Fiona Boland and Tamasine Grimes to the initial study concept, and all of the participating general practitioners and patients.

Contributors: All authors contributed to the conception and design of the study. RMcD, PR, and TF acquired the data. TP, FM, and RMCD analysed the data, and all authors interpreted the data. TP and FM drafted the manuscript, and all authors were involved in critical revision and approval of the final manuscript. The corresponding author attests that all listed authors meet authorship criteria and that no others meeting the criteria have been omitted. TF is the guarantor. Funding: The study was funded by the Health Research Board in Ireland through grant No HRC/2014/1 (TF) and the Spanish Ministry of Economy, Industry, and Competitiveness through grant MTM2016 75351-R (TP). The funders had no role in the design and conduct of the study; collection, management, analysis, and interpretation of the data; preparation, review, or approval of the manuscript; or the decision to submit the manuscript for publication.
Competing interests: All authors have completed the ICMJE uniform disclosure form at www.icmje.org/coi_disclosure.pdf and declare: support for the study as detailed above; no financial relationships with any organisations that might have an interest in the submitted work in the previous three years; and no other relationships or activities that could appear to have influenced the submitted work.

Ethical approval: Ethical approval was obtained from the Irish College of General Practitioners.

Data sharing: No additional data available.

Transparency statement: The lead author affirms that the manuscript is an honest, accurate, and transparent account of the study being reported; that no important aspects of the study have been omitted; and that any discrepancies from the study as planned (and, if relevant, registered) have been explained.

This is an Open Access article distributed in accordance with the Creative Commons Attribution Non Commercial (CC BY-NC 4.0) license, which permits others to distribute, remix, adapt, build upon this work non-commercially, and license their derivative works on different terms, provided the original work is properly cited and the use is non-commercial. See: http://creativecommons. org/licenses/by-nc/4.0/.

1 Qato DM, Wilder J, Schumm LP, Gillet V, Alexander GC. Changes in Prescription and Over-the-Counter Medication and Dietary Supplement Use Among Older Adults in the United States, 2005 vs 2011. JAMA Intern Med 2016;176:473-82. doi:10.1001/ jamainternmed.2015.8581

2 Moriarty F, Hardy C, Bennett K, Smith SM, Fahey T. Trends and interaction of polypharmacy and potentially inappropriate prescribing in primary care over 15 years in Ireland: a repeate cross-sectional study. BMJ Open 2015;5:e008656. doi:10.1136/ bmjopen-2015-008656

3 Mangoni AA, Jackson SH. Age-related changes in pharmacokinetics and pharmacodynamics: basic principles and practical applications. BrJ Clin Pharmacol 2004;57:6-14. doi:10.1046/j.13652125.2003.02007.x

4 Mallet L, Spinewine A, Huang A. The challenge of managing drug interactions in elderly people. Lancet 2007;370:185-91. doi:10.1016/S0140-6736(07)61092-7

5 Petty DR, Zermansky AG, Alldred DP. The scale of repeat prescribing--time for an update. BMC Health Serv Res 2014;14:76. doi:10.1186/1472-6963-14-76

6 Fahey T, Sinclair H. Hospital initiated prescribing in the General Medical Services scheme. Ir Med / 1993:86:122-4

7 Fick D, Waller J, Maclean J, et al. Potentially inappropriate medication use in a Medicare managed care population: Association with higher costs and utilization. / Manag Care Pharm 2001;7:40713doi:10.18553/jmcp.2001.7.5.407.

8 Taché SV, Sönnichsen A, Ashcroft DM. Prevalence of adverse drug events in ambulatory care: a systematic review. Ann Pharmacother 2011;45:977-89. doi:10.1345/aph.1P627

9 Kongkaew C, Noyce PR, Ashcroft DM. Hospital admissions associated with adverse drug reactions: a systematic review of prospective observational studies. Ann Pharmacother 2008:42:1017-25. doi:10.1345/aph.1L037

10 Spinewine A, Schmader KE, Barber N, et al. Appropriate prescribing in elderly people: how well can it be measured and optimised? Lancet 2007;370:173-84. doi:10.1016/S0140-6736(07)61091-5

11 O'Mahony D, O'Sullivan D, Byrne S, O'Connor MN, Ryan C, Gallagher P. STOPP/START criteria for potentially inappropriate prescribing in older people: version 2. Age Ageing 2015;44:213-8. doi:10.1093/ ageing/afu145

12 Hamilton H, Gallagher P, Ryan C, Byrne S, O'Mahony D. Potentially inappropriate medications defined by STOPP criteria and the risk of adverse drug events in older hospitalized patients. Arch Intern Med 2011;171:1013-9. doi:10.1001/archinternmed.2011.215

13 Hill-Taylor B, Sketris I, Hayden J, Byrne S, O'Sullivan D, Christie R. Application of the STOPP/START criteria: a systematic review of the prevalence of potentially inappropriate prescribing in older adults, and evidence of clinical, humanistic and economic impact. J Clin Pharm Ther 2013;38:360-72. doi:10.1111/jcpt.12059

14 Wallace E, McDowell R, Bennett K, Fahey T, Smith SM. Impact of Potentially Inappropriate Prescribing on Adverse Drug Events, Health Related Quality of Life and Emergency Hospital Attendance in Older People Attending General Practice: A Prospective Cohort Study. J Gerontol A Biol Sci Med Sci 2017;72:271-7. doi:10.1093/gerona/ glw140

15 Moriarty F, Bennett K, Cahir C, Kenny RA, Fahey T. Potentially inappropriate prescribing according to STOPP and START and adverse outcomes in community-dwelling older people: a prospective cohort study. Br / Clin Pharmacol 2016;82:849-57. doi:10.1111/ bcp. 12995 
16 Cahir C, Fahey T, Teeling M, Teljeur C, Feely J, Bennett K. Potentially inappropriate prescribing and cost outcomes for older people: a national population study. Br J Clin Pharmacol 2010;69:543-52. doi:10.1111/j.1365-2125.2010.03628.x

17 Vezmar Kovačević S, Simišić M, Stojkov Rudinski S, et al. Potentially inappropriate prescribing in older primary care patients. PLoS One 2014;9:e95536. doi:10.1371/journal.pone.0095536

18 O'Kelly M, Teljeur C, O'Kelly F, Shúilleabháin AN, O'Dowd T. Structure of General Practice in Ireland 1982 - 2015. 2016. https://www.tcd. ie/medicine/public_health_primary_care/assets/pdf/structure-ofgeneral-practice-2016.pdf.

19 Sweeney J, Kearney P, Redmond P, et al. Point of Care Morbidity Coding; a Feasibility Study in Primary Care. Forum J Irish Coll Gen Pract 2017;34:52-4

20 Galvin S, Callan A, Cormican M, et al. Improving antimicrobial prescribing in Irish primary care through electronic data collection and surveillance: a feasibility study. BMC Fam Pract 2015;16:77. doi:10.1186/s12875-015-0280-3

21 von Elm E, Altman DG, Egger M, Pocock SJ, Gøtzsche PC, Vandenbroucke JP, STROBE Initiative. The Strengthening the Reporting of Observational Studies in Epidemiology (STROBE) statement: guidelines for reporting observational studies. PLoS Med 2007:4:e296. doi:10.1371/journal.pmed.0040296

22 Brown JD, Hutchison LC, Li C, Painter JT, Martin BC. Predictive Validity of the Beers and Screening Tool of Older Persons' Potentially Inappropriate Prescriptions (STOPP) Criteria to Detect Adverse Drug Events, Hospitalizations, and Emergency Department Visits in the United States. J Am Geriatr Soc 2016;64:22-30. doi:10.1111/jgs.13884

23 Lau M, Tenney J. Evaluation of Drug-Disease Interactions and Their Association with Unplanned Hospital Readmission Utilizing STOPP Version 2 Criteria. Geriatrics 2017;2:33. doi:10.3390/geriatrics2040033.

24 Hedna K, Hakkarainen KM, Gyllensten H, Jönsson AK, Petzold M, Hägg S. Potentially inappropriate prescribing and adverse drug reactions in the elderly: a population-based study. Eur J Clin Pharmacol 2015;71:1525-33. doi:10.1007/s00228-015-1950-8

25 Walsh KA, O’Riordan D, Kearney PM, Timmons S, Byrne S. Improving the appropriateness of prescribing in older patients: a systematic review and meta-analysis of pharmacists' interventions in secondary care. Age Ageing 2016;45:201-9. doi:10.1093/ageing/afv190

26 Clyne B, Fitzgerald C, Quinlan A, et al. Interventions to address potentially inappropriate prescribing in community-dwelling older adults: a systematic review of randomized controlled trials. / Am Geriatr Soc 2016;64:1210-22. doi:10.1111/igs.14133

27 Sinnott S-J, Bennett K, Cahir C. Pharmacoepidemiology resources in Ireland-an introduction to pharmacy claims data. Eur J Clin Pharmacol 2017:73:1449-55 doi:10.1007/s00228-017-2310-7

28 Charlson ME, Pompei P, Ales KL, MacKenzie CR. A new method of classifying prognostic comorbidity in longitudinal studies: development and validation. / Chronic Dis 1987;40:373-83. doi:10.1016/0021-9681(87)90171-8

29 R Core Team. R: A language and environment for statistical computing. R Foundation for Statistical Computing, 2013.

30 Hadfield ID. MCMC Methods for Multi-Response Generalized Linear Mixed Models: The MCMCglmm R Package. / Stat Softw 2010;33(2):1-22. doi:10.18637/jss.v033.i02

31 Prentice RL, Williams BJ, Peterson AV. On the regression analysis of multivariate failure time data. Biometrika 1981;68:373-9. doi:10.1093/biomet/68.2.373

32 Lin DY, Wei LJ. The Robust Inference for the Cox Proportional Hazards Model. J Am Stat Assoc 1989;84:1074. doi:10.1080/01621459.19 89.10478874

33 Therneau T. Package ‘survival'. 2018. https://cran.r-project.org/web/ packages/survival/survival.pdf.

34 Huntley AL, Johnson R, Purdy S, Valderas JM, Salisbury C. Measures of multimorbidity and morbidity burden for use in primary care and community settings: a systematic review and guide. Ann Fam Med 2012:10:134-41. doi:10.1370/afm.1363

35 D’Agostino RBJr. Propensity score methods for bias reduction in the comparison of a treatment to a non-randomized contro group. Stat Med 1998;17:2265-81. doi:10.1002/(SICI)10970258(19981015)17:19<2265::AID-SIM918>3.0.CO;2-B

36 Ho DE, Imai K, King G, et al. Matching as Nonparametric Preprocessing for Reducing Model Dependence in Parametric Causa Inference. Polit Anal 2007;15:199-236. doi:10.1093/pan/mpl013

37 Almanasreh E, Moles R, Chen TF. The medication reconciliation process and classification of discrepancies: a systematic review. 2016;82:645-58

38 Laroche M-L, Charmes J-P, Nouaille Y, Fourrier A, Merle L. Impact of hospitalisation in an acute medical geriatric unit on potentially inappropriate medication use. Drugs Aging 2006;23:49-59. doi:10.2165/00002512-200623010-00005

39 Gutiérrez-Valencia M, Izquierdo M, Malafarina V, et al. Impact of hospitalization in an acute geriatric unit on polypharmacy and potentially inappropriate prescriptions: A retrospective study. Geriatr Gerontol Int 2017;17:2354-60. doi:10.1111/ggi.13073
40 Rodríguez Del Río E, Perdigones ], Fuentes Ferrer M, et al. [Impact of medium-term outcomes of inappropriate prescribing in older patients discharged from a short stay unit]. Aten Primaria 2018;50:467-76.

41 McMahon CG, Cahir CA, Kenny RA, Bennett K. Inappropriate prescribing in older fallers presenting to an Irish emergency department. Age Ageing 2014;43:44-50. doi:10.1093/ageing/ aft114

42 Blanco-Reina E, García-Merino MR, Ocaña-Riola R, et al. Assessing Potentially Inappropriate Prescribing in Community-Dwelling Older Patients Using the Updated Version of STOPP-START Criteria: A Comparison of Profiles and Prevalences with Respect to the Original Version. PLoS One 2016;11:e0167586. doi:10.1371/journal. pone. 0167586

43 Wauters M, Elseviers M, Vaes B, et al. Too many, too few, or too unsafe? Impact of inappropriate prescribing on mortality, and hospitalization in a cohort of community-dwelling oldest old. Br J Clin Pharmacol 2016;82:1382-92. doi:10.1111/bcp.13055

44 Pardo-Cabello AJ, Manzano-Gamero V, Zamora-Pasadas M, et al. Potentially inappropriate prescribing according to STOPP-2 criteria among patients discharged from Internal Medicine: prevalence, involved drugs and economic cost. Arch Gerontol Geriatr 2018;74:150-4. doi:10.1016/j.archger.2017.10.009

45 Kimura T, Ogura F, Yamamoto K, et al. Potentially inappropriate medications in elderly Japanese patients: effects of pharmacists assessment and intervention based on Screening Tool of Older Persons' Potentially Inappropriate Prescriptions criteria ver.2. J Clin Pharm Ther 2017;42:209-14. doi:10.1111/jcpt.12496

46 Van Craen K, Braes T, Wellens N, et al. The effectiveness of inpatient geriatric evaluation and management units: a systematic review and meta-analysis. J Am Geriatr Soc 2010;58:83-92. doi:10.1111/ j.1532-5415.2009.02621.x

47 Grimes T, Duggan C, Delaney T. Pharmacy services at admission and discharge in adult, acute, public hospitals in Ireland. Int J Pharm Pract 2010;18:346-52. doi:10.1111/j.20427174.2010.00064.x

48 National Patient Experience Survey. Findings of the 2017 inpatient survey. 2018. https://www.patientexperience.ie/app/ uploads/2018/02/NPES-National-Report-2017-WEB.pdf.

49 Moore C, Wisnivesky J, Williams S, McGinn T. Medical errors related to discontinuity of care from an inpatient to an outpatient setting. J Gen Intern Med 2003;18:646-51. doi:10.1046/j.15251497.2003.20722x

50 Kripalani S, LeFevre F, Phillips CO, Williams MV, Basaviah P, Baker DW. Deficits in communication and information transfer between hospital-based and primary care physicians: implications for patient safety and continuity of care. JAMA 2007;297:831-41. doi:10.1001/ jama.297.8.831

51 Witherington EMA, Pirzada OM, Avery AJ. Communication gaps and readmissions to hospital for patients aged 75 years and older: observational study. Qual Saf Health Care 2008;17:71-5. doi:10.1136/qshc.2006.020842

52 Coleman EA. Falling through the cracks: challenges and opportunities for improving transitional care for persons with continuous complex care needs. J Am Geriatr Soc 2003;51:549-55. doi:10.1046/j.1532 5415.2003.51185.x

53 Goodwin N, Dixon A, Poole T, Raleigh V Improving quality of care in general practice: Report of an independent inquiry commissioned by the King's Fund. 2011. https://www.kingsfund.org.uk/sites/default/ files/improving-quality-of-care-general-practice-independent-inquiryreport-kings-fund-march-2011_0.pdf.

54 Le Berre M, Maimon G, Sourial N, Guériton M, Vedel I. Impact of Transitional Care Services for Chronically III Older Patients: A Systematic Evidence Review. J Am Geriatr Soc 2017;65:1597-608. doi:10.1111/jgs.14828

55 Pellegrin KL, Krenk L, Oakes SJ, et al. Reductions in MedicationRelated Hospitalizations in Older Adults with Medication Management by Hospital and Community Pharmacists: A QuasiExperimental Study. J Am Geriatr Soc 2017;65:212-9. doi:10.1111/ jgs.14518

56 Bowden T, Coiera E. The role and benefits of accessing primary care patient records during unscheduled care: a systematic review. BMC Med Inform Decis Mak 2017;17:138. doi:10.1186/s12911-0170523-4

57 Maurice AP, Chan S, Pollard CW, et al. Improving the quality of hospital discharge summaries utilising an electronic prompting system. BMI Qual Improv Rep 2014;3:u200548.w2201. doi:10.1136/bmjquality.u200548.w2201

58 Dreischulte T, Donnan P, Grant A, Hapca A, McCowan C, Guthrie B. Safer Prescribing--A Trial of Education, Informatics, and Financial Incentives. N Engl J Med 2016;374:1053-64. doi:10.1056/ NEJMsa1508955

59 Stocks SJ, Kontopantelis E, Akbarov A, Rodgers S, Avery AJ, Ashcroft DM. Examining variations in prescribing safety in UK general practice: cross sectional study using the Clinical Practice Research Datalink. BMJ 2015;351:h5501. doi:10.1136/bmj.h5501 
60 Anderson K, Stowasser D, Freeman C, Scott I. Prescriber barriers and enablers to minimising potentially inappropriate medications in adults: a systematic review and thematic synthesis. BMJ Open 2014;4:e006544. doi:10.1136/ bmjopen-2014-006544

61 Reeve E, To J, Hendrix I, Shakib S, Roberts MS, Wiese MD. Patient barriers to and enablers of deprescribing: a systematic review. Drugs Aging 2013;30:793-807. doi:10.1007/s40266-013-0106-8

62 Farrell B, Pottie K, Thompson W, et al. Deprescribing proton pump inhibitors: Evidence-based clinical practice guideline. Can Fam Physician 2017;63:354-64

63 Pottie K, Thompson W, Davies S, et al. Deprescribing benzodiazepine receptor agonists: Evidence-based clinical practice guideline. Can Fam Physician 2018;64:339-51.
64 Redmond P, Grimes T, McDonnell R, Boland F, Hughes C, Fahey T. Tackling transitions in patient care: the process of medication reconciliation. Fam Pract 2013:30:483-4. doi:10.1093/fampra/cmt051

65 O'Connor MN, O'Sullivan D, Gallagher PF, Eustace J, Byrne S, O'Mahony D. Prevention of Hospital-Acquired Adverse Drug Reactions in Older People Using Screening Tool of Older Persons' Prescriptions and Screening Tool to Alert to Right Treatment Criteria: A Cluster Randomized Controlled Trial. J Am Geriatr Soc 2016;64:1558-66. doi:10.1111/jgs.14312

66 Grimes TC, Deasy E, Allen A, et al. Collaborative pharmaceutical care in an Irish hospital: uncontrolled before-after study. BMJ Qual Saf 2014;23:574-83. doi:10.1136/bmjqs-2013-002188

\section{Appendix 1-6}

